\title{
Article \\ Cortical Activation in Mental Rotation and the Role of the Corpus Callosum: Observations in Healthy Subjects and Split-Brain Patients
}

\author{
Chiara Pierpaoli ${ }^{1, \dagger}$, Mojgan Ghoushi ${ }^{2}$, Nicoletta Foschi ${ }^{3}$, Simona Lattanzi ${ }^{1}$, Mara Fabri ${ }^{4, *}$ (D) and \\ Gabriele Polonara ${ }^{2}$ (D) \\ 1 Dipartimento di Medicina Sperimentale e Clinica, Università Politecnica delle Marche, 60126 Ancona, Italy; \\ chiara.pierpaoli.124@psypec.it (C.P.); s.lattanzi@univpm.it (S.L.) \\ 2 Dipartimento di Scienze Cliniche Specialistiche e Odontostomatologiche, Università Politecnica Marche, \\ 60126 Ancona, Italy; Mojgan.Ghoushi@ospedaliriuniti.marche.it (M.G.); g.polonara@univpm.it (G.P.) \\ 3 Centro Epilessia, Azienda Ospedaliera-Universitaria Umberto I, 60123 Ancona, Italy; \\ n.foschi@ospedaliriuniti.marche.it \\ 4 Dipartimento di Scienze della Vita e dell'Ambiente, Università Politecnica delle Marche, 60131 Ancona, Italy \\ * Correspondence: m.fabri@univpm.it \\ + Present address: UOST, Inps Casilino-Prenestino, 00155 Roma, Italy.
}

\section{check for}

updates

Citation: Pierpaoli, C.; Ghoushi, M.; Foschi, N.; Lattanzi, S.; Fabri, M.;

Polonara, G. Cortical Activation in

Mental Rotation and the Role of the

Corpus Callosum: Observations in

Healthy Subjects and Split-Brain

Patients. Symmetry 2021, 13, 1953.

https://doi.org/10.3390/

sym13101953

Academic Editor: Enrica

L. Santarcangelo

Received: 13 August 2021

Accepted: 9 October 2021

Published: 16 October 2021

Publisher's Note: MDPI stays neutral with regard to jurisdictional claims in published maps and institutional affiliations.

Copyright: (c) 2021 by the authors. Licensee MDPI, Basel, Switzerland. This article is an open access article distributed under the terms and conditions of the Creative Commons Attribution (CC BY) license (https:/ / creativecommons.org/licenses/by/ $4.0 /)$.

\begin{abstract}
The mental rotation (MR) is an abstract mental operation thanks to which a person imagines rotating an object or a body part to place it in an other position. The ability to perform MR was belived to belong to the right hemisphere for objects, and to the left for one's ownbody images. Mental rotation is considered to be basic for imitation with the anatomical perspective, which in turn is needed for social interactions and learning. Altered imitative performances have been reported in patients with resections or microstructure alterations of the corpus callosum (CC). These patients also display a reduced MR ability compared to control subjects, as shown in a recent behavioral study. The difference was statistically significant, leading us to hypothesize a role of the CC to integrate the two hemispheres' asymmetric functions. The present study was designed to detect, by means of a functional MRI, the cortical activation evoked during an MR task in healthy control subjects and callosotomized patients. The results suggest that performing MR requires activation of opercular cortex and inferior parietal lobule in either hemispheres, and likely the integrity of the CC, thus confirming that the main brain commissure is involved in cognitive functions.
\end{abstract}

Keywords: mental rotation; split-brain patients; imitation; anatomical perspective; corpus callosum; fMRI

\section{Introduction}

The mental rotation (MR) is an abstract mental operation which allows a person imaging to rotate an object or a body part to lead it to a different position [1]. It is a complex cognitive process rooted in real perception, through which mental representations can be recalled from memory, generated and manipulated, even in the absence of environmental input [2]. Occurrence of MR can be referred either to an egocentric or to an object frame [3]: in the first modality the visuo-spatial analysis locates the objects with respect to the observer's point of view [4]; in the latter modality it refers to environmental coordinates [5]. In addition, in egocentric MR tasks, in particular when involving body-related stimuli, individuals should imagine themselves rotating in order to complete the task.

Mental rotation was indagated by measuring, in behavioral experiments, the time to make a judgment about a rotated object, which increases with the amount of rotation necessary to align an object with a referenced one, or with a previously learned template [6-8]. This effect has been observed with both geometric and "abstract" stimuli, and embodied and concrete stimuli [2,9-11]. 
Other studies showed that the MR ability is present in very young children, reaches higher levels during adolescence and declines in the elderly [2,12]. As regards gender differences in MR, the question is still a matter of debate, although several studies indicate a generally higher ability in males $[13,14]$, already present at the age of around 8 years.

Previous studies have suggested that the MR of objects can be proper of the right hemisphere, and MR of body images be proper of the left, although with less evidence $[8,15,16]$. However, since many other studies claim against the right hemisphere dominance for an object's mental rotation, the issue is not solved yet [1,17].

Mental rotation is strictly related to anatomical imitation, as suggested in previous behavioural studies. The imitation is the earliest form of interaction between individuals and it is regarded as the easiest form of social behavior. Imitation assumes an important role in development, motor learning, communication ability and acquisition of social skills. Generally, when invited to imitate gestures executed by a model facing the imitator, subjects can choose between two imitating strategies: a mirror-mode (specular), i.e., using the right limb to copy a spatially matched left limb gesture, or an anatomical-mode, i.e., using the right limb for imitating an anatomically matched right limb gesture by the model. To perform successful anatomical imitation [18], individuals have to make a transformation of the egocentric perspective to the allocentric one [19], which requires an MR operation.

Recent research has investigated imitative behaviour in patients with therapeutic corpus callosum (CC) resection and in healthy adults with an intact CC [20,21]. In these studies the perspective used by participants in imitating intransitive gestures was analyzed: all subjects, when executing spontaneous imitation, mainly used the mirror mode; when instructed to "use the same limb as the model", the control group chose the anatomical mode, but the callosotomy patients still preferred the mirror mode [20,21]. An impairment of the anatomical imitative competence has been evidenced also in psychotic patients in a subsequent study [22], suggesting that individuals with surgical resection, microstructure alteration (as those reported in schizophrenic patients [23,24]), might not be able to execute the MR operation. It has been therefore hypothesized that the different imitative performances of the patients' group with respect to the controls could be due to an impaired capacity for MR, in which the CC seems to have a role. The need of the CC thus suggests that anatomical imitation, and therefore MR ability, on which anatomical imitation is based, are sustained by neural circuits including cortical areas distributed in both hemispheres, with different functions.

Since a close correlation between anatomical imitation and MR could be supposed, in a previous behavioural study, the ability to perform MR was investigated in control healthy subjects, in callosotomized patients and in psychotic patients [25]. The results demonstrate that people with partial or total callosal resection, or with callosal alterations, such as those observed in schizophrenic patients [23,24], exhibited reduced performance in laterality tests with stimuli in third person orientation, suggesting an alteration of the MR mechanism, which could be due to a defective interhemispheric communication (Figure 1) [25]. These results confirmed the need for an interhemispheric cooperation, suggesting therefore a callosal involvement.

The present research was designed with the aim to identify, by functional MRI, the cortical areas activated in control healthy subjects and in callosotomized patients during an MR task with human body pictures, to directly verify whether the cortical activation patterns were similar in intact-brain subjects and in callosotomized patients, and to directly confirm the eventual role of the CC. Attention was also paid to relate the extent of callosal resection with MR ability. A block designed protocol derived from that previously used in the behavioural study [25] was administered. The results showed that performing the MR task evoked the activation of the opercular cortex and of the inferior parietal lobule in either hemisphere, both in controls and in patients. Some patients, however, because of the total or partial lack of the CC, are not able to integrate information between the hemispheres, and therefore are not able to perform MR. Preliminary results have been presented in abstract form [26]. 

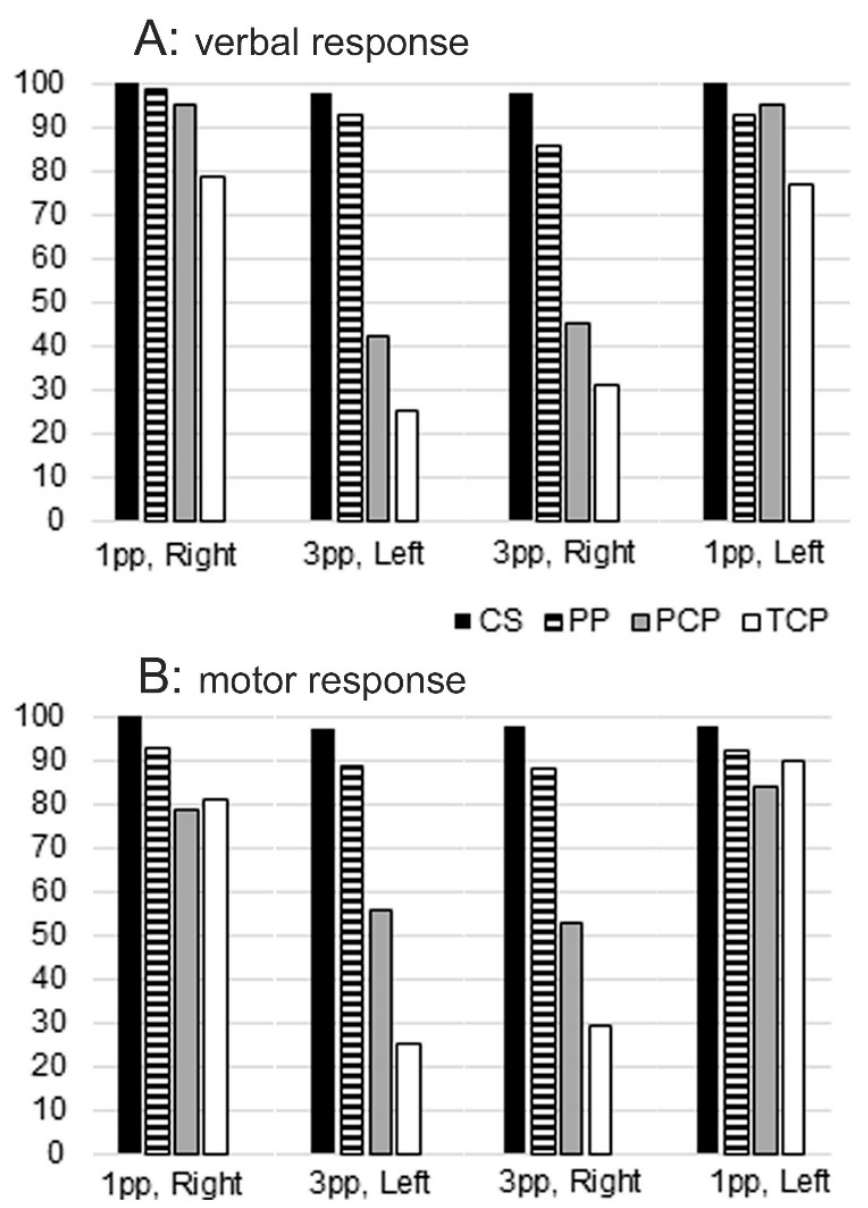

Figure 1. Mental Rotation behavioural performance in healty subjects (black bars), psychotic patients (dashed bars), partially (grey bars) and totally (white bars) callosotomized patients, shown as mean percentage values. (A) verbal session; (B) motor session. In both sessions, patients' performance was a function of the model's position: when she was in first person, all patients made fewer errors. With the model in the third person, callosotomized patients performed worst. CS, control subjects; PCP, partially callosotomized patients; PP, psychotic patients; TCP, totally callosotomized patients; 1pp, first person presentation; 3pp, third person presentation. Modified from [25].

\section{Materlials and Methods}

\subsection{Participants}

Ten healthy adults (control subjecs; aged 24-33 years, mean age 27, SD = 3.37; 5 females; 1 left-handed; Table 1) and 9 callosotomy epileptic patients (aged 37-57 years, mean age 46, $\mathrm{SD}=6.56 ; 3$ females; Figure 2; Table 2) were recruited. Patients had undergone complete or partial surgical resections of the CC to treat medically intractable epilepsy, and followed up at the Neurology Clinic of "Umberto I" Hospital, Ancona. Only patients able to understand the instruction (IQ higher than 70) were recruited. The handedness of all subjects was evaluated using the Edinburgh Inventory [27]; all of them had normal or corrected-tonormal visual acuity. All participants gave their informed consent to the experimental procedure, approved by the Ethics Committee of Università Politecnica delle Marche (Ancona, Italy). 
Table 1. Control healthy subjects participating in the study.

\begin{tabular}{cccccc}
\hline $\begin{array}{c}\text { Control } \\
\text { Subject }\end{array}$ & Gender & Age & $\begin{array}{c}\text { Handedness } \\
\text { (Oldfield Score) }\end{array}$ & fMRI & DTI \\
\hline CS1 & F & 33 & Right (14) & yes & yes \\
\hline CS2 & F & 27 & Right (11) & yes & yes \\
\hline CS3 & M & 24 & Right (23) & yes & yes \\
\hline CS4 & M & 25 & Right (15) & yes & yes \\
\hline CS5 & F & 25 & Right $(20)$ & yes & yes \\
\hline CS6 & M & 27 & Right $(12)$ & yes & yes \\
\hline CS7 & F & 27 & Right $(12)$ & yes & yes \\
\hline CS8 & M & 24 & Right (12) & yes & yes \\
\hline CS9 & M & 33 & Left $(45)$ & Yes & yes \\
\hline CS10 & F & 25 & Right $(15)$ & Yes & yes \\
\hline
\end{tabular}
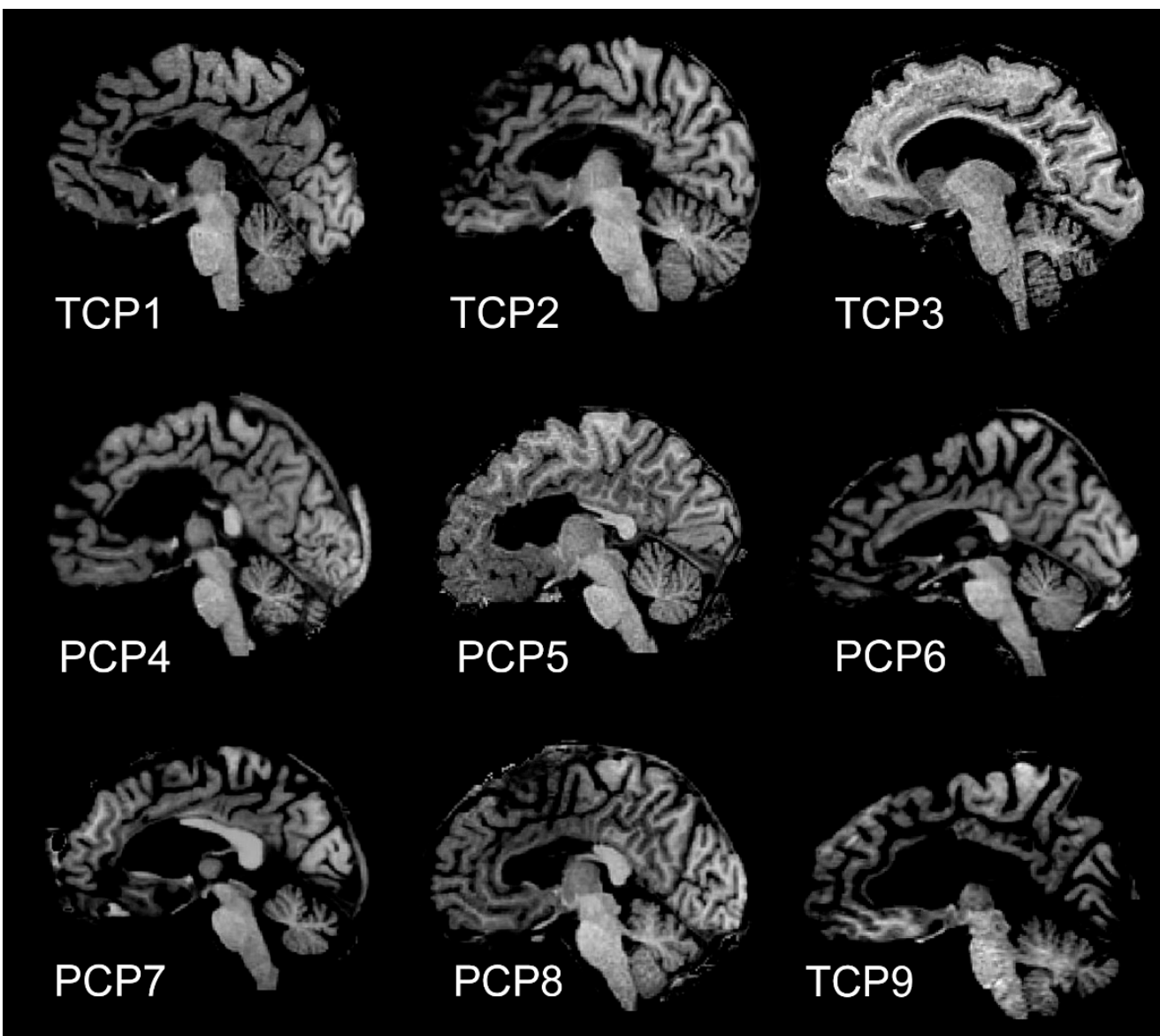

Figure 2. Magnetic resonance images of midsagittal brain slices from the 9 patients participating in the study: 4 of them (TCP1, TCP2, TCP3 and TCP9) underwent total callosotomy, the remaining 5 (PCP4-PCP8) underwent partial anterior callosal resection. 
Table 2. Callosotomized Patients participating in the study.

\begin{tabular}{cccccccc}
\hline Patient & Gender & Age & IQ & $\begin{array}{c}\text { Handedness } \\
\text { (Oldfield } \\
\text { Score) }\end{array}$ & Callosotomy & fMRI & DTI \\
\hline TCP1 & M & 49 & 81 & Right (10) & total & yes & yes \\
\hline TCP2 & M & 39 & 83 & Right (21) & total & yes & yes \\
\hline TCP3 & F & 37 & 70 & Right (10) & total & yes & yes \\
\hline PCP4 & M & 45 & 70 & Right (10) & $\begin{array}{c}\text { partial } \\
\text { anterior }\end{array}$ & yes & yes \\
\hline PCP5 & F & 40 & 70 & Right (10) & $\begin{array}{c}\text { partial } \\
\text { anterior }\end{array}$ & yes & yes \\
\hline PCP6 & M & 49 & 87 & Right (10) & $\begin{array}{c}\text { partial } \\
\text { anterior }\end{array}$ & yes & yes \\
\hline PCP7 & M & 55 & 80 & Right (10) & $\begin{array}{c}\text { partial } \\
\text { anterior }\end{array}$ & yes & yes \\
\hline PCP8 & M & 45 & 70 & Right (10) & $\begin{array}{c}\text { partial } \\
\text { anterior }\end{array}$ & yes & yes \\
\hline TCP9 & F & 57 & 93 & Right (10) & total & yes & yes \\
\hline
\end{tabular}

Since the different performance is often related to the extent of callosotomy [26], the patients' group was split into two, and therefore our study was conducted on three groups of people. Hereafter, the three groups will be reported as Control Subjects (CS), Partially Callosotomized Patients (PCP), and Totally Callosotomized Patients (TCP).

\subsection{Stimuli and Task}

The stimuli were pictures of a model, in the 1st or 3rd person perspective (at $0^{\circ}$ and $180^{\circ}$ ), holding a cup in her right or left hand (4 combinations; Figure 3), which were presented during the fMRI session according to a block-designed protocol alternating a $12 \mathrm{~s}$ period of rest and stimulation.

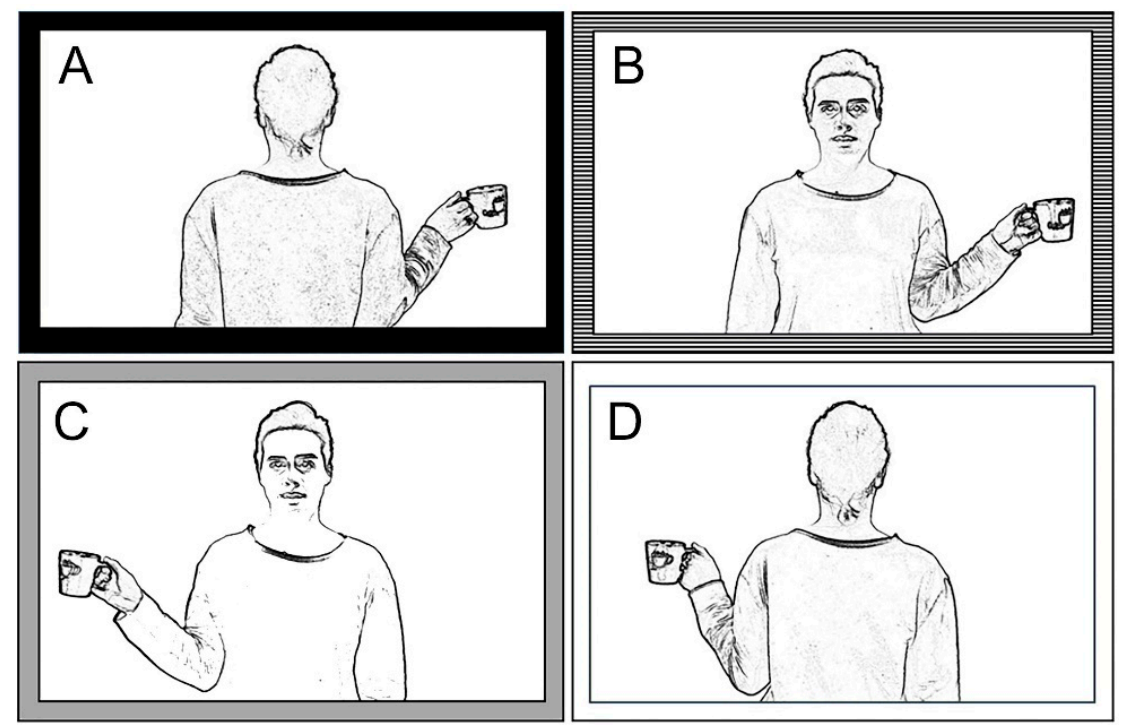

Figure 3. The functional protocol for the evaluation of mental rotation ability is made of 4 images. (A) Model in 1st person, cup in the right hand; (B) model in 3rd person, cup in the left hand; (C) model in 3rd person, cup in the right hand; (D) model in 1st person, cup in the left hand. From [25]. 
All subjects underwent a functional stimulation protocol according to a block designed paradigm. Three $5 \mathrm{~min}$ functional runs were presented to control subjects and to one patient (PCP8), and two runs to the other 8 patients. Each functional run started with a $12 \mathrm{~s}$ rest period (baseline), followed by the first task period; during rest periods, a fixation cross was presented in the center of a grey background.

In each $12 \mathrm{~s}$ task period, one of the pictures described above, depicting the same combination of model and cup position, was flashed 4 times. The same alternation of rest and stimulation periods was administred for all the four different pictures (Figure 4).

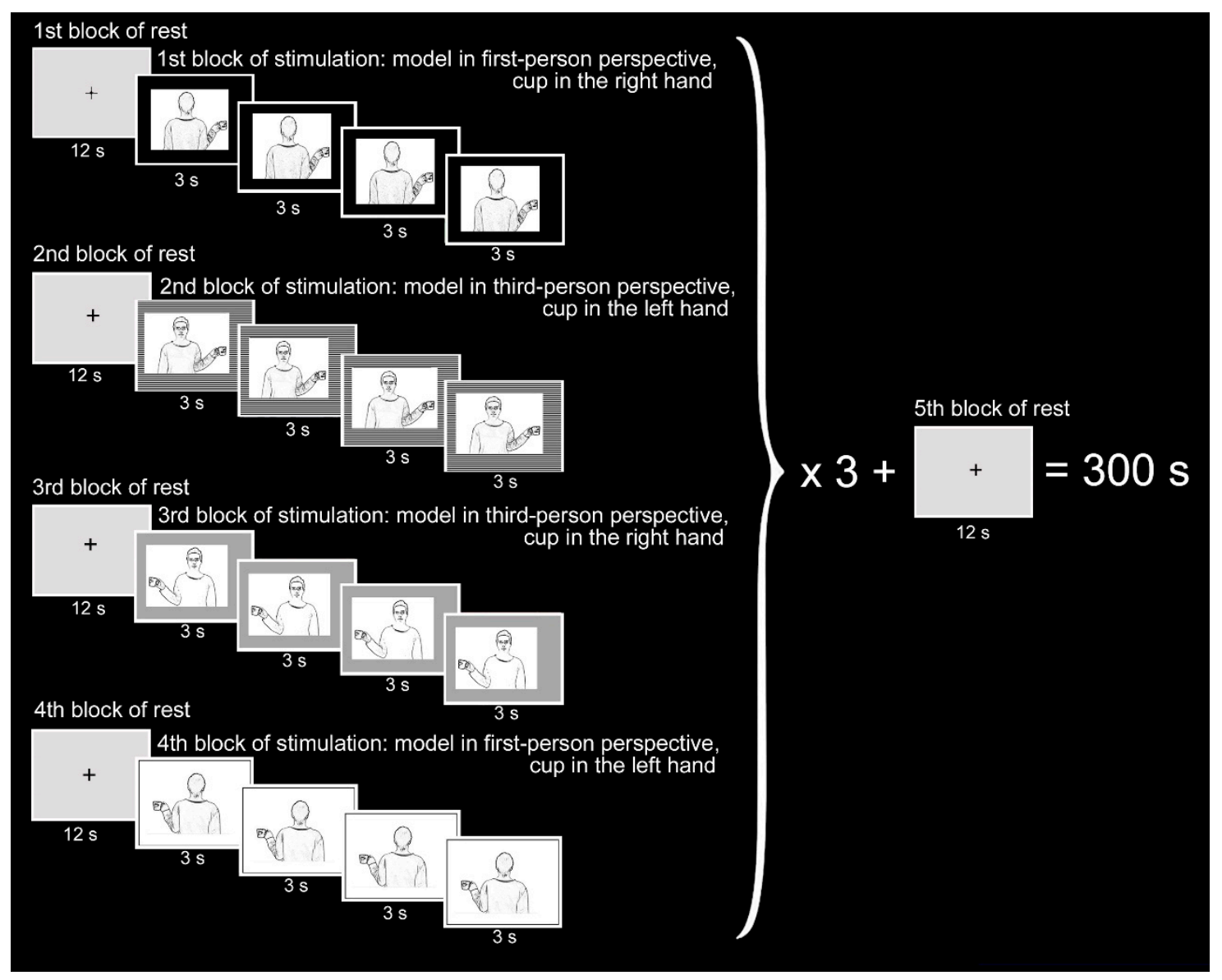

Figure 4. Block designed functional MRI design. The functional design consisted of 2 or 3 identical runs, each lasting 5 min and built of 13 resting periods alternating with 12 stimulation periods. The stimuli were presented in the following order: 1 . model in 1st person with the cup in her right hand (first row); 2 . model in 3rd person with the cup in her left hand (second row); 3. model in 3rd person with the cup in her right hand (third row); 4. model in 1st person with the cup in her left hand (last row). During each stimulation block, the same image was flashed 4 times for $3 \mathrm{~s}$. Blocks were not randomzied.

The following three conditions were investigated:

1. Observation (OBSERVE): the subject was required to carefully observe the pictures. The instruction was: "Please, simply look at the pictures".

2. Hand movement (MOVE): the subject observed the pictures and was requested to move their right (or left) hand, when the cup was in the model's right (or left) hand. The instruction was "Please, as soon as you see the picture, move your right hand if the cup is in the model's right hand, or move your left hand if the cup is in the model's left hand".

3. Thinking (THINK): this condition, being more difficult to control, could be analyzed only in control subjects and in one patient. Subjects were asked to think in which hand of the model the cup was held, in their judice. The instruction was "Please, as soon as you see the picture, think "right" if the cup is in the model's right hand, or think "left" if the cup is in the model's left hand". 
The MR operation was not required to produce a correct response to frames A and D, but it was required to respond correctly to frames B and C.

\subsection{Functional MRI Stimulation Protocol}

Before the scanning sessions, subjects were verbally taught about the experiment. The fMRI sessions consisted of 3 (or 2, see above) functional runs, each composed of 13 resting periods alternating between 12 stimulation periods, and each lasting $12 \mathrm{~s}$. All functional runs started and ended with a resting period; during each stimulation period, the same picture was flashed 4 times, each time for $3 \mathrm{~s}$ (Figure 4 ). The stimulation blocks were presented in the following sequence: model in the 1st person with a cup in the right hand; model in the 3rd person with a cup in the left hand; model in the 3rd person with a cup in the right hand; model in the 1st person with a cup in the left hand (Figure 4).

The pictures were presented through VisualStim Digital glasses (Resonance Technology, Inc., Northridge, CA, USA) worn by each participant before entering the scanner. To reduce head motion artifacts during the data acquisition, we used a custom-head support.

\subsection{Functional MRI Data Acquisition}

Data were collected by means of a $1.5 \mathrm{~T}$ (Signa Excite NV/i CV/i, General Electric Medical System, Milwaukee, WI, USA) provided of $50 \mathrm{mT} / \mathrm{m}$ gradients. Images were transferred to a Unix workstation (General Electric Advantage Windows 4.2) and then to a computer.

Subjects lay in a supine position with the head restrained in a circularly polarized coil, wore 3D glasses, and were invited to avoid any minimal movement.

Through the following 4 steps, an acquisition was performed of:

1. anatomical 3-planes localizer (2D SPGR, TR $120 \mathrm{~ms}$, TE $15 \mathrm{~ms}$, Flip Angle $70^{\circ}$, FOV $23 \times 23 \mathrm{~cm}$, slice thickness $5 \mathrm{~mm}$, Matrix $256 \times 256,1 \mathrm{Nex}$, scan time $31 \mathrm{~s}$ );

2. 3D data set (IR Prep Fast SPGR; TR $15.2 \mathrm{~ms}$, TE $6.9 \mathrm{~ms}$, TI $500 \mathrm{~ms}$, Flip Angle $15^{\circ}$, FOV $29 \times 29 \mathrm{~cm}$, slice thickness $1 \mathrm{~mm}$, Matrix $288 \times 288,1$ Nex, scan time 8:20 min);

3. 20 contiguous 5 -mm-thick axial (or oblique) functional images, single-shot T2* weighted gradient-echo EPI sequence (TR 3000 ms, TE 60 ms, Flip Angle 90 , FOV $28 \times 21 \mathrm{~cm}$, Matrix $96 \times 64,1 \mathrm{Nex}$, scan time 5:12 min);

4. high-resolution axial (or oblique) anatomical images from 20 selected planes (2D SPGR, TR $100 \mathrm{~ms}$, TE $12 \mathrm{~ms}$, Flip Angle 70 ${ }^{\circ}$, FOV $28 \times 21 \mathrm{~cm}$, slice thickness $5 \mathrm{~mm}$, Matrix $256 \times 256,1 \mathrm{Nex}$, scan time 2:25 min) to superimpose functional activation images onto anatomical landmarks, allowing to show blood vessels, possible sources of BOLD signals.

Two thousand functional images (100 per section, 1 image/3 s) were collected during each stimulation cycle from 20 contigous 5 -mm-thick axial sections. Functional images were acquired with the BOLD method. Axial planes were parallel to the AC-PC line, and orthogonal to both sagittal and coronal planes.

\subsection{Functional MRI Data Analysis}

BrainVoyager software package (BrainVoyagerQX, Version 2.3.1.1770, 32-bit, Brain Innovation, Maastricht, The Netherlands, Copyright (C2001-2014 Rainer Goebel) was used for analyzing data (DICOM format) converted into BrainVoyager's internal ".fmr" data format. Corrections for slice scan time was performed; for each participant, each functional volume was aligned to the closest in time anatomical volume. Functional data were superimposed on anatomical brain images, aligned on the AC-PC line, transformed into Talairach [28] space and co-registered with the anatomical. Talairach transformation was performed by means of standard BVQX procedures [29].

An Intensity inhomogeneity correction (IIHC) BrainVoyager tool was applied to perform the standard sequence of preprocessing steps. To minimize the false positive activations and to increase sensitivity to task-related activations, slice scan timing (sinc interpolation based on information about the $\mathrm{TR}=3000 \mathrm{~ms}$ ), 3D correction for motion 
artefacts and temporal filtering were applied; actually, it is impossible to lie completely still during the entire scanning session; in addition, both physiological and physical (scanner related) noise can reduce substantially the power of statistical data analysis; for these reasons, low-pass temporal or spatial filters of preprocessing steps were not applied, as the False Discovery Rate approach used for the specification of an appropriate threshold of statistical maps avoided spatial smoothing.

After the creation of a functional project from measured DICOM files, the original voxel data were stored in STC (slice time course) files.

Functional images of each run from each subject were co-registrated and aligned to $3 \mathrm{D}$ high resolution images and then transformed into Talairach space. In each series, the first two images were discarded to account for signal intensity variations provoked by progressive saturation.

In order to investigate BOLD signals relative to the 3 functional runs (OBSERVE, MOVE and THINK), single-subject contrasts for each run were obtained as follows. First, a Geneal Linear Model (GLM) approach was used to generate statistical parametric maps. In single-subject GLMs, the predictor time courses were convolved with a standard hemodynamic response function (HRF) to take into account the hemodynamic delay. The 4 experimental conditions (i.e., first person/right hand, first person/left hand, third person/right hand, and third person/left hand) were modeled by boxcar waveforms and convolved with the hemodynamic response function. Individual statistical maps was calculated, then the VTC files from multiple subjects underwent to multi-subject analyses, producing statistical maps containing estimated effects (beta values) separately for each subject. Three kinds of multi-subject analyses were performed: one grouping all patients, another grouping separately patients with total callosotomy, and those with partial callosotomy, and the third grouping separately patients who did perform MR and patients who did not. Activation foci were studied by selecting Regions-Of-Interest (ROIs) in different cortical areas in the frontal, parietal and temporal lobes.

By assuming that voxels (or vertices) with similar coordinates in different brains correspond to the same brain regions, raw fMRI time course z-normalization was calculated, to integrate data from multiple subjects into a single GLM analysis, to obtain better comparison across voxels, and to normalize individual runs' variance. When the activation and stimulation time courses were temporally related, activation was assumed to be evoked by the specific predictor.

\section{Results}

This study aimed at defining the cortical areas activated in OBSERVE, MOVE and THINK runs in the three groups of subjects (CS, PCP, and TCP). BOLD signal intensity differences (contrasts) between each run and baseline (OBSERVE > baseline, MOVE > baseline and THINK > baseline) were defined by using a subtraction method. The activation threshold was kept very selective to be sure that all the activations observed were due to the specific task.

Separated analysis was performed for each of the following conditions (c):

$\mathrm{c} 1=$ model in 1st person, cup in the right hand

$\mathrm{c} 2=$ model in 1st person, cup in the left hand

c3 = model in 3rd person, cup in the right hand

c4 = model in 3rd person, cup in the left hand

The description will be provided for all conditions in the text. Data tables for every single condition are also provided.

\section{Control Subjects}

\subsection{Brain Areas Activated during OBSERVE Task}

During OBSERVE run, the contrast task OBSERVE versus baseline produced a map of active voxel clusters, whose Talairach coordinates are shown in Table S3. Multisubject anal- 
ysis generated 39,326 active voxels, analyzed with the FDR statistical approach, selecting a $\mathrm{q}=0.01$ and cluster threshold $=10$ voxels.

In the frontal lobe, the activation of area 6 in the left or right precentral gyrus (PrG; Table S3) in c1 and c2 was observed, respectively; exclusively in c3, an activated focus was present in the right area 10 of the medial frontal gyrus (MFG; Table S3).

No significant activations were observed in the parietal, temporal and insular lobes.

Activation of visual areas of the occipital lobe were observed in both hemispheres (Figure 5B, yellow arrowheads; Table S3) in all conditions: cuneus (Cun; area 17 and area 18), lingual gyrus (LinG; area 18), middle occipital gyrus (MOG; area 19 and area 37).

\subsection{Brain Areas Activated during MOVE Task}

During MOVE run, the contrast task MOVE versus Baseline produced a map of active voxel clusters, whose Talairach coordinates are reported in Table S4. Multisubject analysis generated 38,862 active voxels, to which the FDR statistical approach was applied, with $\mathrm{q}=0.01$ and cluster threshold $=10$ voxels.

In the frontal lobe, activation foci were observed in all conditions in contralateral areas 4 of PrG and 6 of paracentral lobule (Figure 5C, top row; Table S4), bilateral activation was observed in all conditions except c2 (only left) in area 6 of PrG, in all conditions except c3 (only left) in area 6 of MFG (Figure 5C, top row), and in all conditions except $\mathrm{c} 3$ (only right) in area 9 of IFG (Table S4). Activation in the left area 44 of the PrG was also observed in c1 and c4 (Table S4).

In the parietal lobe, activation was consistently observed in all conditions in the contralateral postcentral gyrus (area 2; Figure 5C, top row; Table S4); bilateral activations were observed in the inferior parietal lobule (IPL; area 40) in c3, and in the left side in c1 and c2 (Table S4).

In the temporal lobe, bilateral activation of area 22 in the STG (TPJ) was observed in $\mathrm{c} 1$ and $\mathrm{c} 4$, and absent in the other conditions; unilateral activations were also observed in area 41 (STG and transverse TG), and in area 37 (inferior temporal gyrus, ITG, and fusiform gyrus, FG; Table S4).

In the insular lobe, the activation of area 13 was present in both hemispheres in conditions $\mathrm{c} 1$ and $\mathrm{c} 4$, in the right hemisphere in $\mathrm{c} 2$ and in the left in $\mathrm{c} 3$ (Figure 5C, bottom row, turquoise arrowheads; Table S4).

In the occipital lobe, the activation of visual areas in both hemispheres was observed in all conditions (Figure 5C, bottom row, yellow arrowheads; Table S4): areas 17, 18, and 19.

Activations were also observed in the putamen lentiform nucleus, in the hemisphere contralateral to the moved hand (Figure 5C, bottom row, pink arrows).

\subsection{Brain Areas Activated during THINK Task}

During THINK run, the contrast task THINK versus Baseline produced a map of active voxel clusters, whose Talairach coordinates are reported in Table S5. Multisubject analysis generated 39,806 active voxels, to which the FDR statistical approach was applied, with $\mathrm{q}=0.01$ and cluster threshold $=10$ voxels.

In the frontal lobe, activation foci were observed in contralateral areas 4 (left hemisphere) of PrG in c1 and c3 (Table S5); in area 6 of PrG, in the left (c1) hemisphere, or in both (c3 and c4; Table S5); in c3, activation was also observed in the right IFG, in areas 9, 13 and 45 (Table S5).

In the parietal lobe, the activation of area 7 in the SPL was observed: in the left side in c1, righ side in c2 and bilaterally in c3 and c4 (Figure 5D, top row, pink arrowheads; Table S5). Activation was also observed in the right area 40 in IPL in c4 (Table S5). 

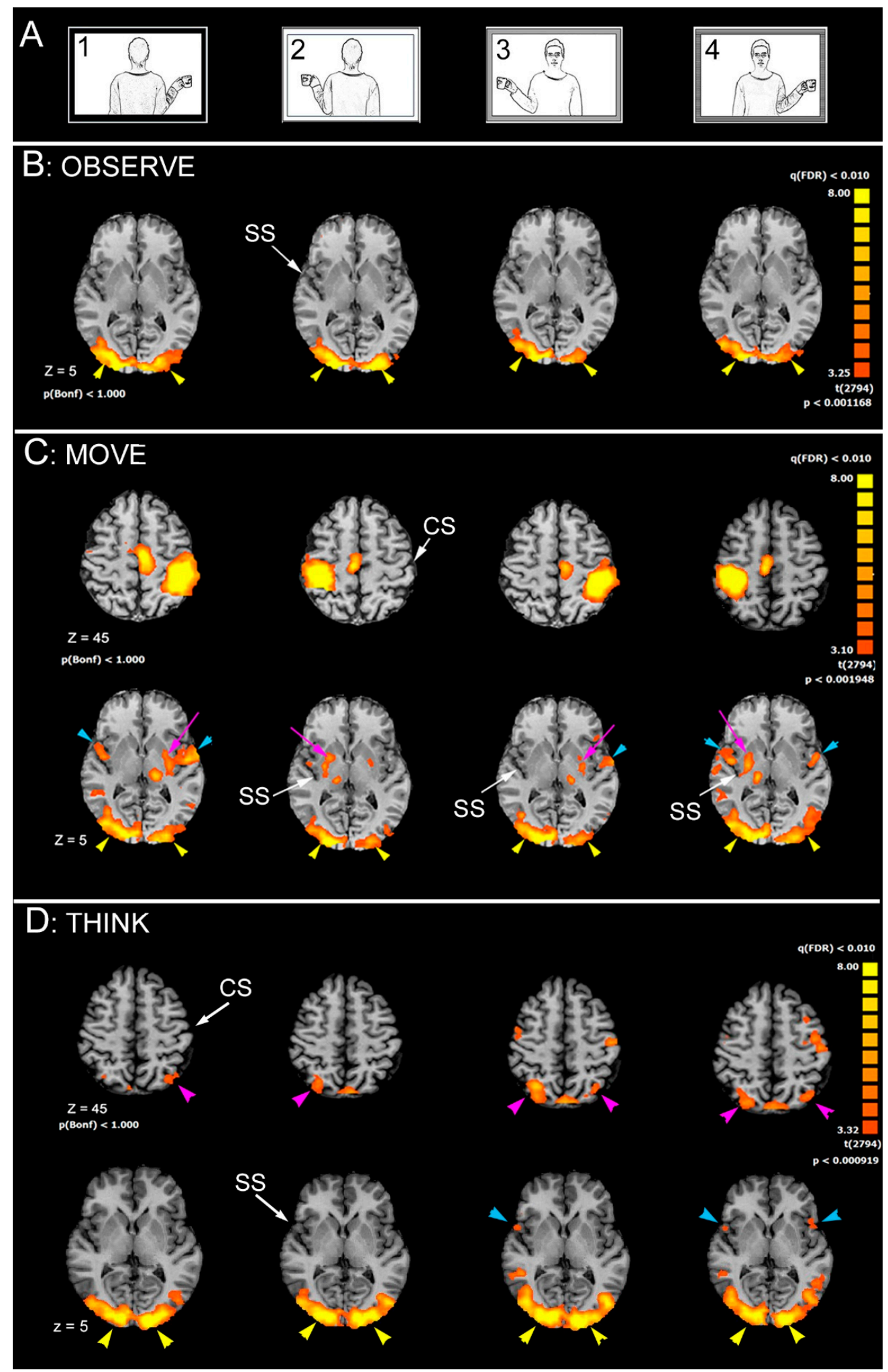

Figure 5. Significant activation in the different runs in control subjects, as obtained from multisubjects analysis. (A) stimulation protocol. (B) OBSERVE condition. (C) MOVE condition. (D) THINK condition. In each condition, axial images are from $z=5$; in the MOVE and THINK condition, also axial images from $z=45$ are shown. CS, central sulcus; SS, sylvian sulcus; according to radiological convention, left hemisphere is on the right. 
In the temporal lobe the unique observed activation was in area 22 in the right STG (TPJ), in c3 and c4 (Table S5).

In the insular lobe, the activation of area 13 was present in the right hemisphere in conditions $\mathrm{c} 3$ and in both henispheres in c4; no activation was observed in c1 and c2 (Table S5).

In the occipital lobe, the activation of visual areas was in both hemispheres in all conditions (Figure 5D, bottom row, yellow arrowheads; Table S5): areas 17, 18, and 19.

\subsection{Callosotomized Patients}

\subsubsection{Brain Areas Activated during OBSERVE Task}

During OBSERVE run, the contrast task OBSERVE versus baseline resulted in map of active voxel clusters. Multisubject analysis generated 31,278 active voxels, to which cluster threshold $=4$ voxels was applied. Since single subject analyses revealed that the activations in all patients were very similar, in this case the description is based on the multisubject analysis from all 9 patients.

In the frontal lobe, the activation was observed in the left area 6 of MFG in c1 only, and in the right area 9 of the PrG.

No significant activations were observed in the parietal, temporal and insular lobes.

In all conditions, activation of visual areas of the occipital lobe were observed in both hemispheres (Figure 6B, yellow arrowheads).

\subsubsection{Brain Areas Activated during MOVE Task}

During MOVE run, the contrast task MOVE versus Baseline resulted in a map of active voxel clusters. Multisubject analysis generated 38,862 active voxels, to which the cluster threshold $=4$ voxels was applied. However, since from single subject analyses high individual variability was evident, the description of results is based on single subjects' activations, showing activations from single significant examples.

In the frontal lobe, activation foci were observed in all conditions in areas 4 of PrG contralateral to the moved hand. In this way, it was possible to verify whether each patient was able to perform the MR. Figure 6C, top row, reports the results from a patient (PCP4) who did not perform MR: activation was observed in area 6 of $\operatorname{PrG}$, and in area 6 of MFG, both in the hemisphere contralateral to the moving hand.

In the parietal lobe, activation was consistently observed in all conditions in the contralateral postcentral gyrus (area 2; Figure 6C, top row); activation was sometimes observed in area 7 in the left hemisphere (not shown).

In the temporal lobe, the activation of area 22 in the STG (TPJ) was sometimes bilateral and sometimes in the left hemisphere.

In the insular lobe, the activation of area 13 was observed in both hemispheres in some cases (Figure 6C, bottom row, turquoise arrowheads), and in the right hemisphere in others (Figure 6D, bottom row, turquoise arrowheads), both in patients who performed MR and in patients who did not.

In the occipital lobe, the activation of visual areas was observed in both hemispheres in all conditions (Figure 6C,D, bottom row, yellow arrowheads).

The THINK task was performed only by patient PCP8, but no significant activation was observed. 

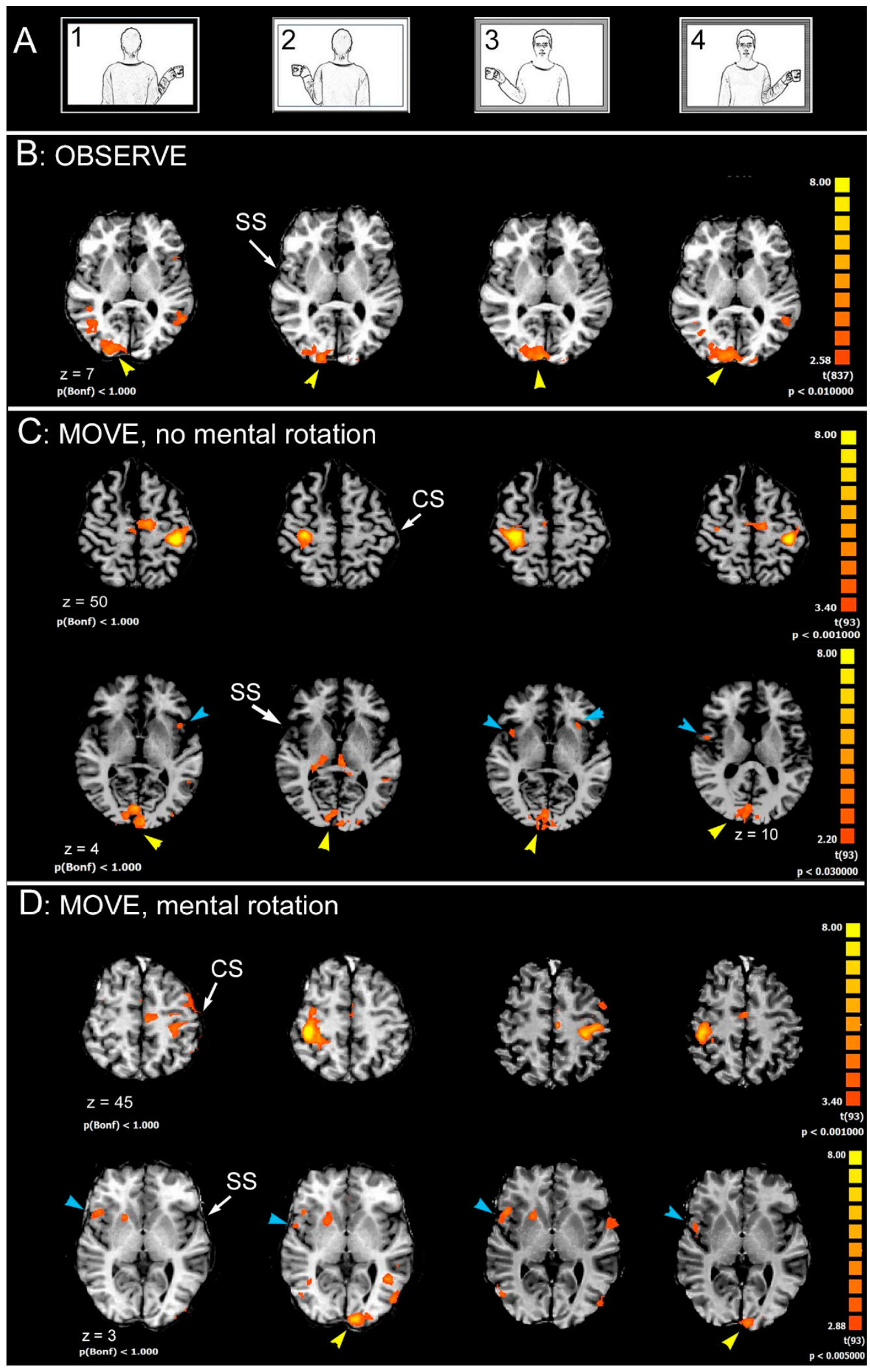

Figure 6. Significant activation in the different runs in callosotomized patients. (A) stimulation protocol. (B) OBSERVE condition $(\mathrm{z}=7)$. (C) MOVE condition: activation from patient PCP4, top row, $\mathrm{Z}=50$ and from patients PCP8, bottom row, $\mathrm{z}=4$, are shown; both of them did not perform mental rotation. (D) MOVE condition: activation from patient PCP5, top row, $Z=45$, and bottom row, $\mathrm{z}=3$, are shown; this patient did perform MR. CS, central sulcus; SS, sylvian sulcus; according to the radiological convention, the left hemisphere is shown on the right. 


\section{Discussion}

The present research investigated the neural circuits underpinning the ability to perform MR of body images in healthy subjects and callosotomized patients. The possibility to study these patients gave us the possibility of directly investigating the role of the CC. The choice to study this group of patients was for the following reasons: 1. callosotomized patients showed an impaired ability for anatomical imitation [21], which likely requires the abstract operation of MR; 2 . an impaired performance in MR in a behavioural contest was reported in our recent paper [25]: most callosotomized patients were not able to perform MR when the model was in 3rd person perspective, likely because of a failure of the neural circuit involved in mental rotation, due to a deficiency in interemispheric communication. The study of Pierpaoli and coworkers [25] indicated the central role of interhemispheric connections in MR, and therefore, strongly suggested that we consider the MR as an asymmetric function.

In the present functional study, participants had to produce two different tasks: in the OBSERVE modality, they simply observed the target stimuli; in the MOVE modality, the subjects must move the hand corresponding to the model's hand holding the cup. In this latter case, the task was laterality judgement in that target stimuli were body images, shown in the first or third perspective, with a cup in either their right or left hand. Therefore, the MOVE task was supposed to recruit MR when responding to front-facing stimuli. In control subjects and in one patient, a third task, THINK, was administered, to evidence only brain regions activated by the MR task.

The results indicated that regions involved in MR in control subjects include lateral area 6 of PrG and area 7 of the SPL in both hemispheres; area 22 of STG (TPJ) in the right hemisphere, and area 13 of insula in both hemispheres. In callosotomized patients, the observed activation pattern was similar, in that it involved lateral area 6 of PrG and area 7 of the SPL, area 22 of STG, and area 13 of insula; however, at variance with control subjects, the activated areas were less consistently found to be in one or another hemishere, and the presence of unilateral or bilateral activation was independent of the ability to perform MR.

These results are essentially in line with previous numerous neuroimaging studies, most of which, like the present one, investigated neural mechanisms underlying MR by means of parity judgment tasks. A network of regions involved in MR was identified, including parietal regions, ventral stream regions (the inferior temporal gyrus, the lateral occipital cortex, and area MT), and higher-order premotor regions (see data and literature in [30]).

In a previous review and meta-analysis of neuroimaging studies [8], the research question was posed, addressing whether MR relies on analog spatial representations or on motor simulation. The meta-analysis was conducted on 32 neuroimaging studies and highlighted a total of 320 activation foci responsive to mental rotation tasks, including areas in the superior parietal, frontal, and infero-temporal cortex. In most of these brain regions, activation was in both hemispheres, except in the parietal cortex, where activity was more consistently observed in the right hemisphere, and in the frontal cortex, where activity was more consistently observed in the left hemisphere [8].

In a following functional study [31], Event-related Potentials (EPs) were calculated from EEG and were continuously acquired during the task. Specifically, in spontaneous and disembodied-self-location tasks, corresponding to the MR for a third person model's presentation of our research, the involvement of the temporo-parietal junction bilaterally was observed, with a right predominant activation; the posterior parietal cortex and the intraparietal sulcus were also activated bilaterally, although more prominently in the left hemisphere. In addition, EP recordings also showed that brain activation was earlier for the back than for the front orientation of the stimuus. This could suggest that body transformation was more difficult in a front-oriented stimulus. During the embodied self-location task, more activated cortical regions were observed in the right hemisphere, as inferior occipital cortex, postcentral gyrus, premotor cortex, dorsolateral prefrontal cortex, anterior temporal cortex; weaker activation was also observed in the temporo- 
parietal junction. Summarizing, these data seem to support an asymmetry in cortical activation: leftward asymmetry when activation was evoked by disembodied self-location, and rightward asymmetry for that evoked by the embodied self-location task (i.e., mirror mode, visuo-spatial match).

A more recent analysis [32] selected $171 \mathrm{fMRI}$ and PET experiments that investigated MR processes, suggesting a modulation of MR networks that were different according to the type of stimulus and strategy used to perform the task (motor imagery-based versus visuo-spatial imagery-based). In particular, they found that the MR of bodily stimuli (hand or body pictures) activated a neural network including many brain areas, some of them in the left hemisphere (i.e., superior frontal gyrus, and inferior parietal lobe), some in the right (i.e., insula), and some in both hemispheres (i.e., superior parietal lobule; for a detailed description see [32]). In addition, by comparing the activation evoked by hand or body stimuli, it was observed that the MR of hands (vs. MR of body stimuli) activated more the left area 6 in the precentral gyrus, and the MR of body stimuli (vs. MR of hands) activated more the left area 18 in the lingual gyrus [32].

All these functional studies $[8,31,32]$ seem to identify the common trend of scientific evidences correlating MR to a sort of hemispheric specialization, despite the different investigation approaches (fMRI, PET, EP) and investigated variables (e.g., instructions, type of stimuli, theoretical definitions). In particular, a left lateralization for the parietal cortex and a right specialization for the frontal regions were evident. In addition, in these studies [31,32], a certain concordance in identifying lateralized activations in motor, sensorimotor and parietal regions during mental rotation was observed: the parietal lobe seems to be more activated in the left hemisphere, whereas the precentral and postcentral regions were in the right.

Mental rotation is a complex cognitive task, involving various processes, such as, for example, discrimination of stimulus orientation, visual imagery, stimulus mental representation, dynamic spatial transformation of images, mental comparison, attentional and working memory, decision-making and implementation of this decision to produce a motor output [32]. Each of these sub-processes could be carried out by specific cortical areas: lateral area 6 appears to code for action at an abstract level, likely in stimulus orientation discrimination [8]; the area in posterior parietal cortex (7 and 40) is involved in visuospatial transformation associated with MR and likely in mental comparison [30]; area 22 (TPJ) would allow a shift from other/self recognition [33], being likely involved in cases of spatial incompatibility [34]; the bilateral activation of the parietal opercula (PO; area 13) was recently reported in healthy subjects also during the anatomical imitation of finger position [35]; since anatomical imitation does require MR, it can be hypothesized that the PO, other than being "implicated in imitation tasks, in coding the body part that executes an action rather the action itself" [35], could also code which part of the body should be considered to receive instruction on whether or not to perform MR. Other studies point to a role for the $\mathrm{PO}$ in the anatomical imitation, either in the right hemisphere [36] or in both [37].

A further discussion about the cortical areas activated in MR tasks in different studies will not here be presented, because our main interest is to demonstrate that activated areas in the two hemispheres should communicate to allow the MR operation. Actually, similar activations were found, although less consistently, also in callosotomized patients, both in those performing MR and in those not; since a big difference between control subjects and patients is the lack, total or partial, of the $\mathrm{CC}$, it can be strongly suggested that MR requires the integrity of the CC, or at least of some of its fibers.

Lateral area 6 communicates with the contralateral hemisphere by sending fibers in the central region of the CC [38]. Interhemispheric fibers from area 7 of SPL cross the isthmus/splenium border. Areas 39-40 of IPL send callosal fibers through the dorsal splenium, and posterior areas 22 and 39 (TPJ) through the ventral splenium [38]. Callosal fibers connecting the PO cortices of the two hemispheres seem to travel across the anterior and central CC body (see [39-41]). Accordingly, patients in which callosal fibres 
from the anterior and/or central body are lacking could display a lower ability of MR performance [25].

It is worthwhile to note that the patients performing MR (PCP5 and PCP6) present the central CC body al least partially spared. This seems to suggest that patients prefer to adopt motor rather than a visual strategies [32]. Actually, a visual strategy would activate visual areas, connected through the CC splenium [38], and a motor strategy would recruit motor cortices, connected through the central CC [38]. Two reasons favour the hypothesis that callosotomized patients more likely used in a motor strategy: the first is that in general, the mental rotation of body images implicitly trigger a motor strategy [16]; the second is that, in general, callosotomized patients need a concrete reference, as a movement, although imagined, could be real. The central CC body seems to be spared in the two other patients (PCP7 and PCP8), who did not perfrom MR. This discrepance could be due to the different level of attention or of instruction understanding, to an interindividual variation of CC morphology and/or fine topography, or to the fact that surgery may have altered the normal morphology of this brain region, and therefore compromised its function [42].

In conclusion, the present results from the control subjects are in line with previous findings, although the exact correspondence in experimental protocols is difficult to assess. In addition, the present research confirms the previous observations that patients with partial or total callosotomy display the worst performance in laterality tests when stimuli are in the third person orientation, indicating a possible alteration of the MR mechanism, likely due to a deficit in the interhemispheric communication [25]. These results are in agreement with previous research reporting the different activation of cortical areas in the two hemispheres, which indicated the need for an interhemispheric cooperation, and therefore for an interhemispheric transfer. The present study therefore further supports the notion that the MR of body images do require interhemispheric communication, strongly suggesting that the CC is involved in a cognitive task. This last observation is in agreement with the known involvement of the CC in a variety of disorders that are currently provoked by genetic alterations, developmental insults, and that are also detectable by alteration of behavior patterns and cognitive and motor performances [43-45].

Supplementary Materials: The following are available online at https:/ /www.mdpi.com/article/10 .3390/sym13101953/s1, Table S3: OBSERVE_Activated areas, Table S4: MOVE—Activated areas, Table S5: THINK-Activated areas.

Author Contributions: Conceptualization, M.F.; methodology, C.P., M.G.; software, C.P., G.P.; validation, N.F., S.L., M.F.; formal analysis, C.P., M.F.; investigation, C.P., G.P., M.G.; resources, N.F., G.P., M.G., M.F.; data curation, C.P., S.L.; writing-original draft preparation, M.F.; writing-review and editing, M.F., C.P., S.L., G.P.; visualization, C.P., S.L.; supervision, S.L., G.P.; project administration, M.F., G.P.; funding acquisition, M.F. All authors have read and agreed to the published version of the manuscript.

Funding: This work was supported by Università Politecnica delle Marche, RSA 2013 (040046_R. SCIENT.A_FABRI_MARA_2013, CUP: I38C 13002630005); RSA 2014 (040046_R.SCIENT.A_FABRI_ MARA_2014, CUP: I32I 14000750005), RSA 2015 (040046_R.SCIENT.A_FABRI_MARA_2015, CUP: I32I 15001420005), PSA 2016 (040046_R.SCIENT.A_2016_FABRI_M_STRATEGICI, CUP I32F 16006480005).

Institutional Review Board Statement: The study was conducted according to the guidelines of the Declaration of Helsinki, and approved by the Comitato Etico Regionale delle Marche, protocol code N. 20150555 OR, date of approval 26 July 2017.

Informed Consent Statement: Informed consent was obtained from all subjects involved in the study.

Data Availability Statement: Data was obtained from Azienda Ospedaliera-Universitaria Umberto I and are available from the authors with the permission of Azienda Ospedaliera-Universitaria Umberto I. 
Acknowledgments: The authors are especially grateful to all volunteers and patients for participating in the study, and to Gabriella Venanzi for her invaluable help in scheduling patients' test sessions. The authors also thank Michele Ombrosi, who performed the initial data analysis for his medical degree dissertation.

Conflicts of Interest: The authors declare no conflict of interest.

\section{References}

1. Corballis, M.C. Mental Rotation and the Right Hemisphere. Brain Lang. 1997, 57, 100-121. [CrossRef]

2. Kosslyn, S.M. Aspects of a cognitive neuroscience of mental imagery. Science 1988, 240, 1621-1626. [CrossRef] [PubMed]

3. Tomasino, B.; Borroni, P.; Isaja, A.; Rumiati, R.I. The role of the primary motor cortex in mental rotation: A TMS study. Cogn. Neuropsychol. 2005, 22, 348-363. [CrossRef] [PubMed]

4. Zacks, J.M.; Michelon, P. Transformations of Visuospatial Images. Behav. Cogn. Neurosci. Rev. 2005, 4, 96-118. [CrossRef] [PubMed]

5. McCloskey, M. Spatial representation in mind and brain. In What Deficits Reveal about the Human Mind/Brain: A Handbook of Cognitive Neuropsychology; Rapp, B., Ed.; Psychology Press: Philadelphia, PA, USA, 2001; pp. 101-132.

6. Shepard, R.N.; Metzler, J. Mental Rotation of Three-Dimensional Objects. Science 1971, 171, 701-703. [CrossRef]

7. Cooper, L.A.; Shepard, R.N. The time required to prepare for a rotated stimulus. Memory Cogn. 1973, 1, 246-250. [CrossRef]

8. Zacks, J.M. Neuroimaging Studies of Mental Rotation: A Meta-analysis and Review. J. Cogn. Neurosci. 2008, 20, 1-19. [CrossRef]

9. Cooper, L.A.; Shepard, R.N. Mental transformation in the identification of left and right hands. J. Exp. Psychol. Hum. Percept. Perform. 1975, 1, 48-56. [CrossRef]

10. Parsons, L.M. Temporal and kinematic properties of motor behavior reflected in mentally simulated action. J. Exp. Psychol. Hum. Percept. Perform. 1994, 20, 709-730. [CrossRef]

11. de Lange, F.P.; Helmich, R.C.; Toni, I. Posture influences motor imagery: An fMRI study. NeuroImage 2006, 33, 609-617. [CrossRef]

12. Jansen, P.; Kaltner, S. Object-based and egocentric mental rotation performance in older adults: The importance of gender differences and motor ability. Aging, Neuropsychol. Cogn. 2013, 21, 296-316. [CrossRef]

13. Linn, M.C.; Petersen, A.C. Emergence and Characterization of Sex Differences in Spatial Ability: A Meta-Analysis. Child Dev. 1985, 56, 1479. [CrossRef]

14. Voyer, D.; Voyer, S.; Bryden, M.P. Magnitude of sex differences in spatial abilities: A meta-analysis and consideration of criticalvariables. Psychol. Bull. 1995, 117, 250. [CrossRef]

15. Parsons, L.M. Superior parietal cortices and varieties of mental rotation. Trends Cogn. Sci. 2003, 7, 515-517. [CrossRef]

16. Tomasino, B.; Vorano, L.; Skrap, M.; Gigli, G.; Rumiati, R.I. Effects of strategies on mental rotation performed by unilateral brain damaged patients. Cortex 2004, 40, 197-199. [CrossRef]

17. Serrati, C.; Finocchi, C.; Calautti, C.; Bruzzone, G.L.; Colucci, M.; Gandolfo, C.; Del Sette, M.; Lantieri, P.B.; Favale, E. Absence of Hemispheric Dominance for Mental Rotation Ability: A Transcranial Doppler Study. Cortex 2000, 36, 415-425. [CrossRef]

18. Nishizawa, H.; Kimura, T.; Goh, A.-C. The effect of different imitation models on the accuracy and speed of imitation of movement. J. Phys. Ther. Sci. 2015, 27, 3417-3420. [CrossRef]

19. Meltzoff, A.N.; Moore, M.K. Imitation, memory, and the representation of persons. Infant Behav. Dev. 1994, 17, 83-99. [CrossRef]

20. Pierpaoli, C.; Ferrante, L.; Manzoni, T.; Fabri, M. Anatomical or mirror mode imitation? A behavioral approach. Arch. Ital. Biol. 2014, 152, 20-31. [PubMed]

21. Pierpaoli, C.; Foschi, N.; Cagnetti, C.; Ferrante, L.; Manzoni, T.; Polonara, G.; Fabri, M. Imitation strategies in callo-sotomeized patients. Arch. Ital. Biol. 2018, 156, 12-26. [PubMed]

22. Sansonetti, R.; Pierpaoli, C.; Ferrante, L.; Fabri, M.; Nardi, B. Imitation strategies in subjects with schizophrenia: A behavioural approach. Arch. Ital. Biol. 2020, 158, 3-16. [CrossRef]

23. Fujino, J.; Takahashi, H.; Miyata, J.; Sugihara, G.; Kubota, M.; Sasamoto, A.; Fujiwara, H.; Aso, T.; Fukuyama, H.; Murai, T. Impaired empathic abilities and reduced white matter integrity in schizophrenia. Prog. Neuro-Psychopharmacol. Biol. Psychiatry 2013, 48, 117-123. [CrossRef] [PubMed]

24. Shahab, S.; Stefanik, L.; Foussias, G.; Lai, M.-C.; Anderson, K.K.; Voineskos, A.N. Sex and Diffusion Tensor Imaging of White Matter in Schizophrenia: A Systematic Review Plus Meta-analysis of the Corpus Callosum. Schizophr. Bull. 2017, 44, $203-221$. [CrossRef] [PubMed]

25. Pierpaoli, C.; Ferrante, L.; Foschi, N.; Lattanzi, S.; Sansonetti, R.; Polonara, G.; Mari, M.; Nardi, B.; Fabri, M. Mental Rotation Ability: Right or Left Hemisphere Competence? What We Can Learn from Callosotomized and Psychotic Patients. Symmetry 2020, 12, 1137. [CrossRef]

26. Fabri, M.; Ombrosi, M.; Goushi, M.; Pierpaoli, C.; Foschi, N.; Lattanzi, S.; Polonara, G. Cortical activation in mental rotation: Observations in healthy subjects and split-brain patients. In Proceedings of the 69th Congress of the Italian Physiological Society, Firenze, Italy, 19-21 September 2018.

27. Oldfield, R.C. The assessment and analysis of handedness: The Edinburgh inventory. Neuropsychologia 1971, 9, 97-113. [CrossRef]

28. Talairach, J.; Turnoux, P. Co-Planar Stereotaxic Atlas of the Human Brain; Thieme Medical Publishers: New York, NY, USA, 2008.

29. Goebel, R. Brainvoyager: A program for analyzing and visualizing functional and structural magnetic resonance data sets. NeuroImage 1996, 3, S604. [CrossRef] 
30. Milivojevic, B.; Hamm, J.P.; Corballis, M.C. Functional Neuroanatomy of Mental Rotation. J. Cogn. Neurosci. 2009, 21, 945-959. [CrossRef]

31. Thirioux, B.; Mercier, M.R.; Jorland, G.; Berthoz, A.; Blanke, O. Mental Imagery of Self-Location during Spontaneous and Active Self-Other Interactions: An Electrical Neuroimaging Study. J. Neurosci. 2010, 30, 7202-7214. [CrossRef]

32. Tomasino, B.; Gremese, M. Effects of Stimulus Type and Strategy on Mental Rotation Network: An Activation Likelihood Estimation Meta-Analysis. Front. Hum. Neurosci. 2016, 9, 693. [CrossRef]

33. Jackson, P.L.; Meltzoff, A.N.; Decety, J. Neural circuits involved in imitation and perspective-taking. NeuroImage 2006, 31, 429-439. [CrossRef]

34. Swoden, S.; Catmur, C. The Role of the Right Temporoparietal Junction in the Control of Imitation. Cereb. Cortex 2015, 25, 1107-1113. [CrossRef]

35. Mengotti, P.; Corradi-Dell'Acqua, C.; Rumiati, R.I. Imitation components in the human brain: An fMRI study. NeuroImage 2012, 59, 1622-1630. [CrossRef] [PubMed]

36. Kubiak, A.; Króliczak, G. Left extrastriate body area is sensitive to the meaning of symbolic gesture: Evidence from fMRI repetition suppression. Sci. Rep. 2016, 6, 31064. [CrossRef] [PubMed]

37. Mengotti, P.; Ticini, L.F.; Waszak, F.; Schütz-Bosbach, S.; Rumiati, R.I. Imitating others' actions: Transcraniam magnetic stimulation on the parietal opercula reveals the processes underlying automatic imitation. Eur. J. Neurosci. 2013, 37, 316-322. [CrossRef] [PubMed]

38. Chao, Y.-P.; Cho, K.-H.; Yeh, C.-H.; Chou, K.-H.; Chen, J.-H.; Lin, C.-P. Probabilistic topography of human corpus callosum using cytoarchitectural parcellation and high angular resolution diffusion imaging tractography. Hum. Brain Mapp. 2009, 30, 3172-3187. [CrossRef] [PubMed]

39. Fabri, M.; Polonara, G. Functional Topography of Human Corpus Callosum: An FMRI Mapping Study. Neural Plast. 2013, 2013, 251308. [CrossRef] [PubMed]

40. Polonara, G.; Mascioli, G.; Foschi, N.; Salvolini, U.; Pierpaoli, C.; Manzoni, T.; Fabri, M.; Barbaresi, P. Further evidence for the topography and connectivity of the corpus callosum: An FMRI study of patients with partial callosal resection. J. Neuroimaging 2014, 25, 465-473. [CrossRef] [PubMed]

41. Mascioli, G.; Berlucchi, G.; Pierpaoli, C.; Salvolini, U.; Barbaresi, P.; Fabri, M.; Polonara, G. Functional MRI cortical activations from unilateral tactile-taste stimulations of the tongue. Physiol. Behav. 2015, 151, 221-229. [CrossRef]

42. Fabri, M.; Polonara, G.; Quattrini, A.; Salvolini, U.; Del Pesce, M.; Manzoni, T. Role of the corpus callosum in the somatosensory activation of the ipsilateral cerebral cortex: An fMRI study of callosotomized patients. Eur. J. Neurosci. 1999, 11, 3983-3994. [CrossRef]

43. Frazier, T.W.; Hardan, A.Y. A Meta-Analysis of the Corpus Callosum in Autism. Biol. Psychiatry 2009, 66, 935-941. [CrossRef]

44. Paul, L.K. Developmental malformation of the corpus callosum: A review of typical callosal development and examples of developmental disorders with callosal involveme. J. Neurodevelop. Disord. 2011, 3, 3-27. [CrossRef]

45. Frederiksen, K.S. Corpus callosum in aging and dementia. Dan. Med. J. 2013, 60, B4721. 